\title{
Ploidy and Genome Size in Lilac Species, Cultivars, and Interploid Hybrids
}

\author{
Jason D. Lattier ${ }^{1}$ and Ryan N. Contreras ${ }^{2}$ \\ Department of Horticulture, 4017 Agriculture and Life Sciences Building, Oregon State University, \\ Corvallis, OR 97331-7304
}

\begin{abstract}
AdDitional INDEX wORDS. flow cytometry, cytology, triploid, aneuploidy, unreduced gametes
Abstract. Genome size variation can be used to investigate biodiversity, genome evolution, and taxonomic relationships among related taxa. Plant breeders use genome size variation to identify parents useful for breeding sterile or improved ornamentals. Lilacs (Syringa) are deciduous trees and shrubs valued for their fragrant spring and summer flowers. The genus is divided into six series: Syringa (Vulgares), Pinnatifoliae, Ligustrae, Ligustrina, Pubescentes, and Villosae. Reports conflict on genome evolution, base chromosome number, and polyploidy in lilac. The purpose of this study was to investigate genome size and ploidy variation across a diverse collection. Flow cytometry was used to estimate monoploid (1Cx) and holoploid (2C) genome sizes in series, species, cultivars, and seedlings from parents with three ploidy combinations: $2 x \times 2 x, 2 x \times 3 x$, and $3 x \times 2 x$. Pollen diameter was measured to investigate the frequency of unreduced gametes in diploid and triploid Syringa vulgaris cultivars. Three triploids of S. vulgaris were observed: 'Aucubaefolia', 'Agincourt Beauty', and 'President Grévy'. Across taxa, significant variations in $1 \mathrm{C} x$ genome size were discovered. The smallest and largest values were found in the interspecific hybrids $S$. $\times$ laciniata $(1.32 \pm 0.04 \mathrm{pg})$ and $S$. $\times$ hyacinthiflora 'Old Glory' $(1.78 \pm 0.05)$, both of which are in series Syringa. Series Syringa $(1.68 \pm 0.02 \mathrm{pg})$ had a significantly larger $1 \mathrm{C} x$ genome size than the other series. No significant differences were found within series Pubescentes $(1.47 \pm 0.01 \mathrm{pg})$, Villosae $(1.55 \pm 0.02 \mathrm{pg})$, Ligustrina $(1.49 \pm 0.05 \mathrm{pg})$, and Pinnatifoliae (1.52 $\pm 0.02 \mathrm{pg})$. For $S$. vulgaris crosses, no significant variation in $2 \mathrm{C}$ genome size was discovered in $2 x \times 2 x$ crosses. Interploid crosses between 'Blue Skies' $(2 x)$ and 'President Grévy' $(3 x)$ produced an aneuploid population with variable $2 \mathrm{C}$ genome sizes ranging from $3.41 \pm 0.03$ to $4.35 \pm 0.03 \mathrm{pg}$. Only one viable seedling was recovered from a cross combination between 'President Grévy' $(3 x)$ and 'Sensation' $(2 x)$. This seedling had a larger 2C genome size $(5.65 \pm \mathbf{0 . 0 2} \mathbf{~ p g})$ than either parent and the largest $2 \mathrm{C}$ genome size currently reported in lilac. 'Sensation' produced $8.5 \%$ unreduced pollen, which we inferred was responsible for the increased genome size. No unreduced pollen was discovered in the other diploids examined. Increased ploidy may provide a mechanism for recovering progeny from incompatible taxa in lilac breeding.
\end{abstract}

Genome size variation can be used to investigate biodiversity, taxonomic relationships, and genome evolution among related taxa (Greilhuber, 1998; Rounsaville and Ranney, 2010; Shearer and Ranney, 2013; Zonneveld and Duncan, 2010; Zonneveld et al., 2005). Studies on genome evolution focus on large, structural changes in sequence or fluctuations in genome size resulting from natural phenomena including polyploidy, chromosome fission/fusion, and interploid hybridization (Soltis and Soltis, 2012). Genome size variation can also be used by plant breeders to identify parents for wide hybrids among parent taxa. Interspecific hybrids have been shown to have genome sizes intermediate between their parents in other woody ornamentals such as Cornus (Shearer and Ranney, 2013) and Magnolia (Parris et al., 2010). When combining genome sizes with their corresponding chromosome counts, genome size data can be used to discover ploidy variation among related taxa (Contreras et al., 2013; Lattier, 2016; Parris et al., 2010; Shearer and Ranney, 2013). Polyploidy, or whole genome duplication, is a driving force in evolution and occurs naturally through somatic mutations in meristematic cells and through unreduced gametes (Harlan and deWet, 1975; Ranney, 2006). There are two broad categories of polyploidy; autopolyploidy

Received for publication 14 June 2017. Accepted for publication 13 July 2017. This research was funded in part by the Oregon Department of Agriculture.

We acknowledge the assistance of Mara Friddle, Kim Shearer, and Aleen Haddad in this research.

${ }^{1}$ Graduate Research Assistant.

${ }^{2}$ Corresponding author. E-mail: ryan.contreras@oregonstate.edu. is the duplication of a single genome, whereas allopolyploidy is the combination of two or more different genomes and an associated duplication event (Chen and $\mathrm{Ni}, 2006$ ).

The identification and induction of polyploidy can be valuable tools for plant breeding. Irregular meiosis in gametes can result in sterility, whereas "gigas" effects of somatic cells can result in thicker, glossier cuticles, enlarged flowers, or enlarged fruit (Ranney, 2006). In addition, polyploids have been used to overcome interploid hybridization barriers (Ranney, 2006) and to restore fertility in wide hybrids of ornamentals such as Rhododendron 'Fragrant Affinity' and $\times$ Chitalpa tashkentensis (Contreras et al., 2007; Olsen et al., 2006).

Syringa is a genus of deciduous, woody trees, and shrubs grown primarily for their heavy spring and summer blooms of fragrant flowers. Syringa comprised 21-28 species that are part of the monophyletic subfamily Oleoideae in family Oleaceae and are closely allied with Ligustrum (Li et al., 2002; Wallander and Albert, 2000). Recent taxonomic studies divide the genus into six series: Syringa, Pinnatifoliae, Ligustrae, Ligustrina, Pubescentes, and Villosae (Li et al., 2012). Most species are native to eastern Asia while two species, $S$. vulgaris and $S$. josikaea, are native to southeastern Europe (Kim and Jansen, 1998). Most cultivar development over centuries of breeding has focused on improvements of common lilac (S. vulgaris) within series Syringa.

Lilacs have proven to be important ornamental crops, yet little is known about how nuclear genome varies among series, species, hybrids, and cultivars. A survey of genome 
size (C-value) and ploidy level within Syringa would contribute to the call for a global census of angiosperm $\mathrm{C}$-values (Galbraith et al., 2011). Although genome sequencing is a powerful tool for studying gene function, $\mathrm{C}$-values calculated from sequencing data tend to underestimate true genome size (relative to flow cytometry) because of misassembly and the inability to sequence through repetitive regions of the genome (Bennett and Leitch, 2011). Flow cytometry measurements of genome size have proven useful for the identification of species, hybrids, polyploids, and polyploid series (Galbraith et al., 2011).

In genera such as lilac with a long history of breeding and cultivation, variation in genome size and chromosome number can occur from interspecific hybridization, unreduced gametes, and the induction of autopolyploids. Interspecific hybridization has been a valuable tool for producing many new cultivars of lilac (Table 1). Two reports on genome size estimates in lilac focused on two European species, S. vulgaris and S. josikaea. Siljak-Yakovlev et al. (2010) reported $S$. vulgaris to have a $2 \mathrm{C}$ genome size of $2.4 \mathrm{pg}$ based on propidium iodide flow cytometry. Olszewska and Osiecka (1984) reported S. josikaea to have a 2C genome size of $2.6 \mathrm{pg}$ based on Feulgen cytophotometry. Despite the paucity of genome size estimates in lilac, much effort has been dedicated to studying chromosome number variation in lilac and the Oleaceae.

Phylogenetic analysis has determined the ancestral state of the Oleaceae to be diploid (Taylor, 1945). Cyto-taxonomy divides the Oleaceae into two groups according to basic chromosome number with the first group consisting of Mendora $(x=11)$, Jasminum $(x=13)$, Fontanesia $(x=13)$, Forsythia $(x=14)$, and Abeliophyllum $(x=14)$. The second group (originally designated as subfamily Oleoideae) consists of genera with $x=23$, including Olea, Syringa, Ligustrum, Fraxinus, Osmanthus, Forestiera, Phillyrea, Osmarea, and Chionanthus (Taylor, 1945). Lilacs are primarily diploids with basic chromosome numbers reported at $x=22,23$, or 24 (Darlington and Wylie, 1956). Sax (1930) reported the "fundamental" chromosome number in lilac to be $x=12$ and hypothesized that ancestral polyploidization of an $x=11$ or $x=12$ cytotype was responsible for the variation in chromosome numbers, with the $x=23$ cytotype resulting from the loss of a pair of chromosomes. By contrast, Taylor (1945) reported that most wild-type lilac specimens are $x=23$ cytotypes, not $x=22$ or $x=24$. The prevalence and stability of the $x=23$ cytotype throughout the Oleaceae, illustrated by Taylor (1945) and Stebbins (1940), indicates that the $x=23$ cytotype likely predates other cytotypes

Table 1. Previously named interspecific hybrids in lilac including their series and pedigree information (Fiala and Vrugtman, 2008).

\begin{tabular}{|c|c|c|c|c|}
\hline Interspecific hybrid & $q$ parent & q series & ऽ parent & $\widehat{o}$ series \\
\hline Syringa $\times$ chinensis & S. protolaciniata & Syringa & S. vulgaris & Syringa \\
\hline Syringa $\times$ diversifolia ${ }^{\mathrm{z}}$ & S. pinnatifolia & Pinnatifoliae & S. oblata ssp. oblata & Syringa \\
\hline Syringa $\times$ henryi & S. josikaea & Villosae & S. villosa & Villosae \\
\hline Syringa $\times$ hyacinthiflora & S. oblata & Syringa & S. vulgaris & Syringa \\
\hline Syringa $\times$ josiflexa & S. josikaea & Villosae & S. reflexa & Villosae \\
\hline Syringa $\times$ laciniata & Unknown & Unknown & Unknown & Unknown \\
\hline Syringa $\times$ nanceiana & S. ×henryi & Villosae & S. sweginzowii & Villosae \\
\hline Syringa $\times$ persica & Unknown & Unknown & Unknown & Unknown \\
\hline Syringa $\times$ prestoniae & S. villosa & Villosae & S. komarowii & Villosae \\
\hline Syringa $\times$ swegiflexa & S. komarowii & Villosae & S. sweginzowii & Villosae \\
\hline
\end{tabular}

${ }^{\mathrm{z}}$ The single named interspecific hybrid resulting from an interseries cross in lilacs. in Syringa and originated as the result of allopolyploidy between ancestral Oleaceae taxa of two cytotypes, $x=11$ and $x=12$. Therefore, the variation in chromosome number observed in common lilac is likely the result of aneuploidy over centuries of plant collection and wide hybridization.

Aside from theories of ancestral allopolyploidy, no reports exist to confirm polyploidy in wild or cultivated lilac populations. In addition, no reports of natural polyploidy exist for the closely related genus Ligustrum. However, natural polyploidy has been discovered in other related genera in Oleaceae. Taylor (1945) reported tetraploids in Mendora, tetraploids and triploids in Jasminum, tetraploids and hexaploids in Fraxinus, and hexaploids in Osmanthus. In white ash (Fraxinus), the tetraploid $F$. smallii and hexaploids, such as $F$. biltmoreana and $F$. profunda, are hypothesized to have allopolyploid origins (Miller, 1955; Nesom, 2010; Santamour, 1962).

Early efforts producing artificial polyploids in lilac were reported to be successful. In the middle of the 20th century, Karl Sax produced colchicine-induced autopolyploids of $S$. vulgaris at the Arnold Arboretum (Fiala and Vrugtman, 2008). Fiala reportedly produced tetraploid forms of $S$. julianae, $S$. komarowii, S. ×prestoniae, S. wolfi, S. yunnanensis, S. vulgaris, S. oblata, and $S$. ×hyacinthiflora (Fiala and Vrugtman, 2008).

Despite these previous reports of induced polyploidy, no cytological evidence exists to support these claims. Lilacs have been bred for centuries, yet polyploid lilac breeding remains a largely unexplored field (Fiala and Vrugtman, 2008). Few modern studies have confirmed successful induction of autopolyploid lilacs. Rose et al. (2000) created mixoploid and tetraploid lilacs from colchicine-treated cuttings of an interseries hybrid, $S$. vulgaris $\times S$. pinnatifolia. Rothleutner (2014) recovered diploids, mixoploids, tetraploids, and octoploids from oryzalin-treated seedlings of $S$. reticulata cultivars. Both Rose et al. (2000) and Rothleutner (2014) used flow cytometry to confirm autopolyploids. In many crops, hybridization between tetraploid and diploid populations has been useful for creating sterile triploid progeny due to meiotic irregularities. Where some fertility exists in triploids, they can provide an important bridge in wide crosses and their range of gametes can be used in the production of high copy number polyploids such as tetraploids, pentaploids, and hexaploids (Wang et al., 2010). Aneuploid progeny have been produced in other woody plants through diploid-triploid hybridization including Pyrus (Phillips et al., 2016), Ulmus (Santamour, 1971), and Populus (Wang et al., 2010).

The purpose of this study was to explore the genome size, ploidy variation, and presence of unreduced gametes in a diverse collection of lilacs including representative species and cultivars from five lilac series and interploid hybrids in series Syringa.

\section{Methods and Materials}

Plant material. Lilac taxa were acquired from gardens, arboreta, and nurseries. Representative taxa were obtained from five of the six series within genus Syringa including Syringa, Pubescentes, Villosae, Ligustrina, and 
Pinnatifoliae. Series Ligustrae, which includes genus Ligustrum nested within genus Syringa (Li et al., 2012), was not included. Included in our study were 54 total taxa including species, cultivars, and hybrids (Table 2). Species and subspecies designations are based on current taxonomy (Chen et al., 2009; Li et al., 2012). In lilac, cultivar or trademark names are rarely interchangeable with only one becoming the market name that commonly identifies a cultivar. As a reference, cultivar and trademark names are reported (Table 2), but for simplicity, only market names (cultivar or trademark) are used hereafter. A subset of hybrids was created among selected parent taxa to investigate seedling genome size variation in the following parent cytotype combinations: $3 x \times 2 x, 2 x \times 3 x$, and $2 x \times 2 x$.

FLOw CYTOMETRY. Flow cytometry was used to assess holoploid (2C) genome size (relative to an internal standard) for each individual taxon in the lilac collection at Oregon State University. References to genome size and ploidy follow the terminology proposed by Greilhuber et al. (2005). For each taxon, three vegetative buds or three young, fully expanded leaves were collected to represent a random sample of nuclei. Included with each taxon was a leaf sample of the internal standard of known genome size, Pisum sativum 'Ctirad' $[2 \mathrm{C}=8.76$ pg (Bai et al., 2012; Greilhuber et al., 2007)]. Each sample was prepared by cochopping 1-2 $\mathrm{cm}^{2}$ of tissue from both lilac and an internal standard ( $P$. sativum 'Ctirad') with a razor in a polystyrene petri dish containing $400 \mu \mathrm{L}$ of nuclei extraction buffer solution (Cystain Ultraviolet Precise P Nuclei Extraction Buffer; Sysmex, Görlitz, Germany). A buffer containing the chopped leaf tissue was passed through a $30-\mu \mathrm{m}$ gauze filter (Partec Celltrics, Münster, Germany) into a 3.5-mL plastic tube (Sarstedt Ag \& Co., Nümbrecht, Germany). Next, $1.6 \mathrm{~mL}$ of fluorochrome stain (4',6-diamidino-2-phenylindole) was added to the nuclei suspension (Cystain Ultraviolet Precise P Staining Buffer; Partec). All samples were analyzed using a flow cytometer (CyFlow Ploidy Analyzer; Partec). A minimum of 3000 nuclei were analyzed per sample with an average $\mathrm{CV}$ for each fluorescence histogram under 10. Relative $2 \mathrm{C}$ genome size was calculated as:

$$
\begin{aligned}
2 \mathrm{C}= & \text { DNA content of standard } \\
& \times \frac{\text { mean fluorescence value of sample }}{\text { mean fluorescence value of standard }}
\end{aligned}
$$

Monoploid (1Cx) genome size was calculated using ploidy determined using root tip microscopy (described below) as:

$$
1 \mathrm{C} x=\frac{2 \mathrm{C}}{\text { ploidy }}
$$

The estimations of chromosome numbers in aneuploid seedlings were based on a genome size estimate of a single, theoretical chromosome $(0.061 \mathrm{pg})$ calculated as:

Genome size of a single chromosome

$$
=\frac{\text { Polyploid parent }(2 \mathrm{C} \mathrm{pg})-\text { Diploid parent }(2 \mathrm{C} \mathrm{pg})}{\text { difference in chromosome number }}
$$

Genome size variation in parent taxa and progeny resulting from different cytotype combinations were investigated using flow cytometry. Histogram figures from flow cytometry (.fcs files) were produced using open-source Cytospec software from Purdue University Cytometry Laboratories (2014).
Chromosome counts. Chromosome counts were performed on several taxa representing four series: Syringa, Pubescentes, Villosae, and Ligustrina. An improved protocol for preparing root tips for chromosome counts (Lattier et al., 2017) was followed for lilac, with lilac root tips digested by enzyme for 2-3 h. Chromosomes were visualized (Axio imager.A1; Zeiss, Thornwood, NY) and imaged (AxioCam 105 Color; Zeiss) at different focal distances and layered to increase resolution for each photomicrograph. Focus-stacking was performed using the Auto Blend feature in Photoshop CC 2015.5.1 (Adobe Systems, San Jose, CA). A minimum of 15 resolved cells were investigated per taxa.

Pollen CYTOLOGY. From a previous study on cross-compatibility (Lattier and Contreras, 2017), a single seedling from an interploid cross was discovered to have a larger genome size than either parent suggesting that unreduced gametes were present in one parent. Therefore, both parents (S. vulgaris 'President Grévy' and $S$. vulgaris 'Sensation') as well as two other randomly selected taxa (S. vulgaris 'Ludwig Spaeth' and S. vulgaris 'Miss Ellen Willmott') were screened for unreduced pollen grains. At anthesis, fresh flowers were collected from each plant, and pollen was dusted onto microscope slides. Three slides were prepared and measured for each cultivar. To each slide, a single drop of $2 \%$ acetocarmine was added and then a cover slip was added. All slides were screened for stained pollen grains at a magnification of $\times 100$ on a light microscope (Axio imager.A1). Single fields of view were randomly captured (AxioCam 105 Color) across each microscope slide and all stained pollen grains were measured using the line measurement tool in AxioVision SE64 4.9.1 (Zeiss). A total of 5381 pollen grains were measured in the four cultivars of $S$. vulgaris. Figures of reduced and unreduced pollen grains were focus-stacked to increase resolution using the Auto Blend feature of Photoshop CC 2015.5.1. To estimate unreduced pollen grains, the following equation was used:

$$
\text { Volume of a sphere }=\frac{4}{3} \pi r^{3}
$$

As the volume of a sphere (pollen grain) doubles, the diameter increases by $26 \%$. Therefore, any pollen grains with a diameter greater than $26 \%$ of the average for each taxa was scored as an unreduced pollen grain. Percent unreduced pollen grains were calculated as:

$$
\text { Percent unreduced pollen }=\frac{\text { unreduced pollen grains }}{\text { total pollen grains }} \times 100
$$

Statistical analysis. Statistical analyses were performed using SAS Studio (version 3.6; SAS Institute, Cary, NC). Monoploid genome sizes were analyzed with the PROC GLM. Mean genome size averages for each individual taxon were separated using Tukey's honestly significant difference test $(\alpha=0.05)$. Genome size averages for each series were generated from an average of individual taxa means. Least squares means for each series were separated using a Tukey-Kramer test for unequal sample sizes $(\alpha=0.05)$. Least squares means were also separated for pollen diameter measurements of four cultivars of $S$. vulgaris using a Tukey-Kramer test for unequal sample sizes $(\alpha=0.05)$.

\section{Results and Discussion}

Genome sizes. Holoploid 2C genome sizes ranged from $2.64 \pm 0.08 \mathrm{pg}$ in $S$. Xlaciniata to $4.94 \pm 0.06 \mathrm{pg}$ in $S$. vulgaris 
Table 2. Taxonomic, trademark, accession, and source information for Syringa source material used in the current study.

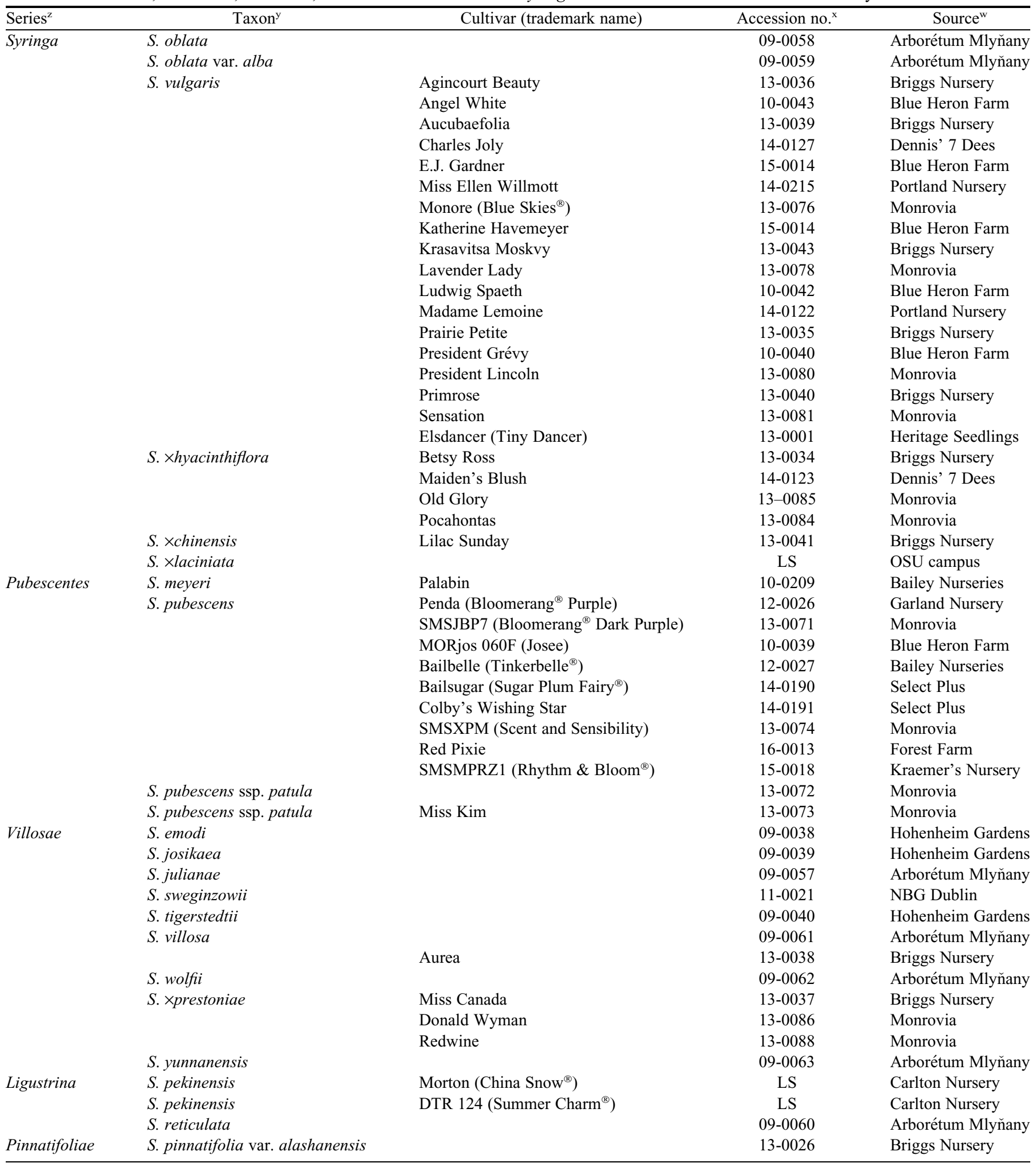

${ }^{\mathrm{z}}$ Series designation based on Li et al. (2012).

${ }^{\mathrm{y}}$ Individual taxon in Syringa based on (Li et al., 2012) and revisions (Chen et al., 2009).

${ }^{\mathrm{x}}$ Accession number in research population; $\mathrm{LS}=$ nonaccessioned leaf samples for flow cytometry.

${ }^{\mathrm{w}}$ Container plants, seeds, and leaf samples collected from the following sources: Arborétum Mlyñany (Slepcany, Slovakia), Bailey Nurseries (Yamhill, OR), Blue Heron Farm (Corvallis, OR), Briggs Nursery (Elma, WA), Carlton Plants (Dayton, OR), Dennis' 7 Dees Landscaping \& Garden Centers (Portland, OR), Garland Nursery (Corvallis, OR), Heritage Seedlings \& Liners (Salem, OR), Hohenheim Gardens (Stuttgart, Germany), Kraemer's Nursery (McMinnville, OR), Monrovia (Dayton, OR), National Botanic Gardens [NBG Dublin (Glasnevin, Ireland)]; Oregon State University [OSU campus (Corvallis, OR)], Portland Nursery (Portland, OR), Select Plus International Lilac Nursery (Mascouche, Canada). 
'Aucubaefolia'. All 2C relative genome sizes were larger than the two previously reported genome sizes of European lilacs (Olszewska and Osiecka, 1984; Siljak-Yakovlev et al., 2010). Previous reports have shown similar variation due to different binding properties of fluorochrome stains (Lattier, 2016; Parris et al., 2010). Only three taxa of S. vulgaris, nested within series Syringa, had a 2C relative genome size larger than $4.00 \mathrm{pg}$, including $S$. vulgaris 'Aucubaefolia' (4.94 \pm $0.06 \mathrm{pg})$, S. vulgaris 'Agincourt Beauty' (4.90 $\pm 0.03 \mathrm{pg})$, and S. vulgaris 'President Grévy' (4.85 \pm 0.00 pg). Chromosome counts of $S$. vulgaris 'Aucubaefolia' revealed this group to be triploids (Fig. 1). The presence of triploids in our collection supports early reports of polyploid induction experiments and interploid hybridization (Fiala and Vrugtman, 2008) but surprisingly, no tetraploids were observed. All other root tip cells investigated were diploid, including $S$. ×hyacinthiflora 'Maiden's Blush', S. Xhyacinthiflora 'Old Glory', $S$. ×prestoniae 'Miss Canada', S. reticulata, and S. pubescens Bloomerang ${ }^{\circledR}$ Purple (Fig. 1). Chromosome counts in the current study provided no evidence for base chromosome number other than $x=23$ (Fig. 1), in contrast to previous reports that varied from $x=22$ to 24 (Darlington and Wylie, 1956).

Significant differences were found among taxa for $1 \mathrm{C} x$ genome size $(P<0.0001)$. Values ranged from $1.32 \pm 0.04 \mathrm{pg}$ in $S$. ×laciniata to $1.78 \pm 0.05 \mathrm{pg}$ in $S$. ×hyacinthiflora 'Old Glory' (Table 3). Series Syringa had a significantly larger average $1 \mathrm{C} x$ genome size $(1.68 \pm 0.02 \mathrm{pg})$ than the other four series investigated (Table 3 ). There were no significant differences among series Pubescentes (1.47 $\pm 0.01 \mathrm{pg})$, Villosae
$(1.55 \pm 0.02 \mathrm{pg})$, Ligustrina $(1.49 \pm 0.05 \mathrm{pg})$, and Pinnatifoliae $(1.52 \pm 0.02 \mathrm{pg})$ (Table 3$)$.

Within series Syringa, S. ×laciniata had a significantly smaller genome size compared with other tested taxa in series Syringa. No reports exist on the pedigree of $S$. $\times$ laciniata (Table 1), although Fiala and Vrugtman (2008) hypothesize it to a cross of the Afghan lilac, $S$. protolaciniata, and another unknown parent. Syringa $\times$ laciniata has a heavily dissected leaf, much like $S$. pinnatifolia, while $S$. protolaciniata produces heterophyllous leaves with margins varying from lobed to entire (Fiala and Vrugtman, 2008; Green, 1995). In addition, the only other heavily dissected lilac, $S$. pinnatifolia, has also proven to be the only species successfully used in interseries crosses (Pringle, 1981). If $S$. Xlaciniata is from an interseries hybridization, then aneuploidy concomitant with wide hybridization could explain the significant reduction in genome size compared with other taxa in series Syringa. Further chromosome counts need to be performed on this hybrid.

Most $1 \mathrm{C} x$ genome sizes within series Syringa were above $1.60 \mathrm{pg}$ (Table 3). Although S. oblata is native to Asia and $S$. vulgaris is native to southeastern Europe, their different geographical origins are not reflected in significant genome size variation. Wild-type $S$. oblata and the white-flowered, S. oblata var. alba, both had a $1 \mathrm{C} x$ genome size of $1.73 \pm 0.03 \mathrm{pg}$ (Table $3)$. The smallest and largest monoploid genome sizes in $S$. vulgaris were from two white, double-flowered taxa, S. vulgaris 'Miss Ellen Willmott' $(1.61 \pm 0.01 \mathrm{pg})$ and $S$. vulgaris 'Madame Lemoine' (1.76 $\pm 0.05 \mathrm{pg}$ ) (Table 3$)$. Taxa representing hybrids between $S$. oblata and $S$. vulgaris had a monoploid genome size range from $S$. $\times$ hyacinthiflora 'Betsy Ross' $(1.70 \pm$ $0.02 \mathrm{pg}$ ) to $S$. Xhyacinthiflora 'Old Glory' (1.78 $\pm 0.05 \mathrm{pg}$ ); however, there were no differences among the four hybrid cultivars included (Table 3). One additional interspecific hybrid, $S$. $\times$ chinensis (1.74 \pm $0.07 \mathrm{pg}$ ), representing a cross between $S$. protolaciniata and $S$. vulgaris was found to have a similar $1 \mathrm{Cx}$ genome size to $S$. vulgaris (Table 3 ).

Within series Pubescentes, most $1 \mathrm{C} x$ genome sizes were below $1.50 \mathrm{pg}$ and there were no significant differences among the 12 taxa included. The smallest genome size was S. pubescens Rhythm \& Bloom ${ }^{\circledR}$ $(1.43 \pm 0.01 \mathrm{pg})$, whereas the largest was in $S$. pubescens ssp. patula 'Miss Kim' (1.54 \pm 0.01 pg). Within series Villosae, most $1 \mathrm{C} x$ genome sizes were above $1.50 \mathrm{pg}$ and ranged from $S$. tigerstedtii $(1.38 \pm 0.01 \mathrm{pg})$ to $S$. villosa 'Aurea' $(1.62 \pm 0.03 \mathrm{pg})$ (Table 3). Syringa villosa exhibited a $1 \mathrm{C} x$ genome size similar to cultivars of $S$. ×prestoniae, which has S. villosa along with $S$. komarowii in its pedigree (Table 1). Syringa tigerstedtii had a significantly smaller $1 \mathrm{C} x$ genome size compared with all other taxa except $S$. xprestoniae 'Donald Wyman' $(1.50 \pm 0.00 \mathrm{pg})$
Fig. 1. Stained chromosomes in root tip cells of six accessions of Syringa. Photomicrographs viewed at $\times 1000$ with scale bar at $1 \mu \mathrm{m}$ : (A) Triploid $(2 n=3 x=69)$ S. vulgaris 'Aucubaefolia'; (B) diploid $(2 n=2 x=46)$ S. $\times$ hyacinthiflora 'Maiden's Blush'; $(\mathbf{C})$ diploid $(2 n=2 x=46)$ S. ×hyacinthiflora 'Old Glory'; (D) diploid $(2 n=2 x=46) S$. $\times$ prestoniae 'Miss Canada'; $(\mathbf{E})$ diploid $(2 n=2 x=46)$ S. reticulata; $(\mathbf{F})$ diploid $(2 n=2 x=46)$ S. pubescens Bloomerang ${ }^{\circledR}$ Purple. 
Table 3. Ploidy and relative genome size in Syringa determined using flow cytometry analysis of DAPI-stained nuclei with Pisum sativum 'Ctirad' $(8.76 \mathrm{pg} / 2 \mathrm{C})$ as an internal standard.

\begin{tabular}{|c|c|c|c|c|}
\hline Series $^{z}$ & $1 \mathrm{C} x$ genome size $[\text { mean } \pm \mathrm{SE}(\mathrm{pg})]^{\mathrm{y}}$ & $\operatorname{Taxa}^{\mathrm{x}}$ & Ploidy & $1 \mathrm{C} x$ genome size $[\text { mean } \pm \mathrm{SE}(\mathrm{pg})]^{\mathrm{w}}$ \\
\hline \multirow[t]{26}{*}{ Syringa } & \multirow[t]{26}{*}{$1.68 \pm 0.02 \mathrm{a}$} & S. oblata & $2 x$ & $1.73 \pm 0.03 \mathrm{a}-\mathrm{d}$ \\
\hline & & S. oblata var. alba & $2 x$ & $1.73 \pm 0.03 \mathrm{a}-\mathrm{c}$ \\
\hline & & S. vulgaris 'Agincourt Beauty' & $3 x$ & $1.63 \pm 0.01 \mathrm{a}-\mathrm{m}$ \\
\hline & & S. vulgaris 'Angel White' & $2 x$ & $1.67 \pm 0.05 \mathrm{a}-\mathrm{k}$ \\
\hline & & S. vulgaris 'Aucubaefolia' & $3 x^{\mathrm{v}}$ & $1.65 \pm 0.02 \mathrm{a}-1$ \\
\hline & & S. vulgaris Blue Skies ${ }^{\circledR}$ & $2 x$ & $1.72 \pm 0.02 \mathrm{a}-\mathrm{e}$ \\
\hline & & S. vulgaris 'Charles Joly' & $2 x$ & $1.69 \pm 0.02 \mathrm{a}-\mathrm{h}$ \\
\hline & & S. vulgaris 'E.J. Gardner' & $2 x$ & $1.66 \pm 0.01 \mathrm{a}-\mathrm{k}$ \\
\hline & & S. vulgaris 'Miss Ellen Willmott' & $2 x$ & $1.61 \pm 0.01 \mathrm{~b}-\mathrm{o}$ \\
\hline & & S. vulgaris 'Katherine Havemeyer' & $2 x$ & $1.71 \pm 0.03 \mathrm{a}-\mathrm{e}$ \\
\hline & & S. vulgaris 'Krasavitsa Moskvy' & $2 x$ & $1.70 \pm 0.00 \mathrm{a}-\mathrm{h}$ \\
\hline & & S. vulgaris 'Lavender Lady' & $2 x$ & $1.69 \pm 0.02 \mathrm{a}-\mathrm{h}$ \\
\hline & & S. vulgaris 'Ludwig Spaeth' & $2 x$ & $1.74 \pm 0.03 \mathrm{a}-\mathrm{c}$ \\
\hline & & S. vulgaris 'Madame Lemoine' & $2 x$ & $1.76 \pm 0.05 \mathrm{a}-\mathrm{b}$ \\
\hline & & S. vulgaris 'Prairie Petite' & $2 x$ & $1.69 \pm 0.03 \mathrm{a}-\mathrm{i}$ \\
\hline & & S. vulgaris 'President Grévy' & $3 x$ & $1.62 \pm 0.00 \mathrm{~b}-\mathrm{O}$ \\
\hline & & S. vulgaris 'President Lincoln' & $2 x$ & $1.73 \pm 0.01 \mathrm{a}-\mathrm{d}$ \\
\hline & & S. vulgaris 'Primrose' & $2 x$ & $1.68 \pm 0.01 \mathrm{a}-\mathrm{j}$ \\
\hline & & S. vulgaris 'Sensation' & $2 x$ & $1.67 \pm 0.02 \mathrm{a}-\mathrm{k}$ \\
\hline & & S. vulgaris Tiny Dancer & $2 x$ & $1.71 \pm 0.02 \mathrm{a}-\mathrm{g}$ \\
\hline & & S. ×hyacinthiflora 'Betsy Ross' & $2 x$ & $1.70 \pm 0.02 \mathrm{a}-\mathrm{g}$ \\
\hline & & S. ×hyacinthiflora 'Maiden's Blush' & $2 x^{\mathrm{v}}$ & $1.74 \pm 0.07 \mathrm{a}-\mathrm{c}$ \\
\hline & & S. $\times$ hyacinthiflora 'Old Glory' & $2 x^{\mathrm{v}}$ & $1.78 \pm 0.05 \mathrm{a}$ \\
\hline & & S. $\times$ hyacinthiflora 'Pocahontas' & $2 x$ & $1.75 \pm 0.02 \mathrm{a}-\mathrm{b}$ \\
\hline & & S. $\times$ chinensis 'Lilac Sunday' & $2 x$ & $1.74 \pm 0.07 \mathrm{a}-\mathrm{c}$ \\
\hline & & S. $\times$ laciniata & $2 x$ & $1.32 \pm 0.04 \mathrm{u}$ \\
\hline \multirow[t]{12}{*}{ Pubescentes } & \multirow[t]{12}{*}{$1.47 \pm 0.01 \mathrm{~b}$} & S. meyeri 'Palabin' & $2 x$ & $1.47 \pm 0.03 \mathrm{n}-\mathrm{u}$ \\
\hline & & S. pubescens Bloomerang ${ }^{\circledR}$ Purple & $2 x^{\mathrm{v}}$ & $1.46 \pm 0.02 \mathrm{o}-\mathrm{u}$ \\
\hline & & S. pubescens Bloomerang ${ }^{\circledR}$ Dark Purple & $2 x$ & $1.49 \pm 0.041-\mathrm{t}$ \\
\hline & & S. pubescens 'Colby's Wishing Star' & $2 x$ & $1.52 \pm 0.02 \mathrm{k}-\mathrm{t}$ \\
\hline & & S. pubescens Josee & $2 x$ & $1.45 \pm 0.04 \mathrm{p}-\mathrm{u}$ \\
\hline & & S. pubescens 'Red Pixie' & $2 x$ & $1.49 \pm 0.011-\mathrm{t}$ \\
\hline & & S. pubescens Rhythm \& Bloom ${ }^{\circledR}$ & $2 x$ & $1.43 \pm 0.01 \mathrm{q}-\mathrm{u}$ \\
\hline & & S. pubescens Scent and Sensibility & $2 x$ & $1.47 \pm 0.00 \mathrm{n}-\mathrm{u}$ \\
\hline & & S. pubescens Sugar Plum Fairy ${ }^{\circledR}$ & $2 x$ & $1.47 \pm 0.03 \mathrm{n}-\mathrm{u}$ \\
\hline & & S. pubescens Tinkerbelle ${ }^{\circledR}$ & $2 x$ & $1.40 \pm 0.01 \mathrm{~s}-\mathrm{u}$ \\
\hline & & S. pubescens ssp. patula & $2 x$ & $1.48 \pm 0.01 \mathrm{~m}-\mathrm{t}$ \\
\hline & & S. pubescens ssp. patula 'Miss Kim' & $2 x$ & $1.54 \pm 0.01 \mathrm{~h}-\mathrm{s}$ \\
\hline \multirow[t]{12}{*}{ Villosae } & \multirow[t]{12}{*}{$1.55 \pm 0.02 \mathrm{~b}$} & S. emodii & $2 x$ & $1.55 \pm 0.01 \mathrm{~g}-\mathrm{s}$ \\
\hline & & S. josikaea & $2 x$ & $1.57 \pm 0.01 \mathrm{e}-\mathrm{r}$ \\
\hline & & S. julianae & $2 x$ & $1.59 \pm 0.03 \mathrm{c}-\mathrm{p}$ \\
\hline & & S. sweginzowii & $2 x$ & $1.55 \pm 0.02 \mathrm{~g}-\mathrm{s}$ \\
\hline & & S. tigerstedtii & $2 x$ & $1.38 \pm 0.01 \mathrm{t}-\mathrm{u}$ \\
\hline & & S. villosa & $2 x$ & $1.56 \pm 0.03 \mathrm{f}-\mathrm{r}$ \\
\hline & & S. villosa 'Aurea' & $2 x$ & $1.62 \pm 0.03 \mathrm{~b}-\mathrm{n}$ \\
\hline & & S. wolfii & $2 x$ & $1.57 \pm 0.02 \mathrm{~d}-\mathrm{q}$ \\
\hline & & S. ×prestoniae 'Donald Wyman' & $2 x$ & $1.50 \pm 0.001-\mathrm{t}$ \\
\hline & & S. ×prestoniae 'Miss Canada' & $2 x^{v}$ & $1.61 \pm 0.03 \mathrm{~b}-\mathrm{o}$ \\
\hline & & S. ×prestoniae 'Redwine' & $2 x$ & $1.53 \pm 0.02 \mathrm{i}-\mathrm{t}$ \\
\hline & & S. yunnanensis & $2 x$ & $1.58 \pm 0.01 \mathrm{c}-\mathrm{q}$ \\
\hline \multirow[t]{3}{*}{ Ligustrina } & \multirow[t]{3}{*}{$1.49 \pm 0.05 \mathrm{~b}$} & S. pekinensis China Snow ${ }^{\circledR}$ & $2 x$ & $1.41 \pm 0.02 \mathrm{r}-\mathrm{u}$ \\
\hline & & S. pekinensis Summer Charm ${ }^{\circledR}$ & $2 x$ & $1.47 \pm 0.03 \mathrm{n}-\mathrm{u}$ \\
\hline & & S. reticulata & $2 x^{\mathrm{v}}$ & $1.59 \pm 0.03 \mathrm{c}-\mathrm{q}$ \\
\hline Pinnatifoliae & $1.52 \pm 0.02 \mathrm{~b} \ddagger$ & S. pinnatifolia var. alashanensis & $2 x$ & $1.52 \pm 0.02 \mathrm{j}-\mathrm{t}$ \\
\hline
\end{tabular}

${ }^{\mathrm{z}}$ Series designation based on phylogeny by Li et al. (2012).

${ }^{\mathrm{y}}$ Series means based on average of taxa means; letters represent Tukey-Kramer test for unequal sample sizes $(\alpha=0.05)$; $\downarrow=$ three samples of same accession were used to calculate mean.

${ }^{\mathrm{x}}$ Taxa grouped within series; species and market name (cultivar or trademark) presented.

${ }^{\text {w}}$ Means separated using Tukey's honest significant test (HSD) $(\alpha=0.05)$; means followed by same letter are not significantly different; dash between letters indicate complete series of letters; minimum significant difference $=0.158$.

Ploidy confirmed with root tip cytology. 
and 'Redwine' (1.53 $\pm 0.02 \mathrm{pg})$ (Table 3$)$. However, no other significant differences were found throughout series Villosae, even though this diverse series was the most species-rich in the collection.

Within the tree lilacs (series Ligustrina) only two species, $S$. reticulata and $S$. pekinensis, and few cultivars exist. Green and Chang (1995) previously reported only one species, S. reticulata, with other species circumscribed to the rank of subspecies. The $1 \mathrm{C} x$ genome sizes of $S$. pekinensis China $\mathrm{Snow}^{\circledR}$ and Summer Charm ${ }^{\circledR}$ were not significantly different. However, a significant difference was detected between $S$. pekinensis China Snow ${ }^{\circledR}(1.41 \pm 0.02 \mathrm{pg})$ and $S$. reticulata $(1.59 \pm 0.03$ pg) (Table 3). Within the monotypic series Pinnatifoliae, $S$. pinnatifolia var. alashanensis had a $1 \mathrm{Cx}$ genome size of $1.52 \pm 0.02$ (Table 3).

Hybrid GeNOME SIZES. Based on genome size estimates of parent taxa, hybrids from a previous cross-compatibility study on lilacs (Lattier and Contreras, 2017) were evaluated for genome size variation. As a seed parent, more than 800 flowers of the triploid S. vulgaris 'President Grévy' were pollinated in intraspecific crosses with four different diploids: $S$. vulgaris 'Tiny Dancer', S. vulgaris 'Angel White', S. vulgaris 'President Lincoln', and S. vulgaris 'Sensation' (Lattier and Contreras, 2017). Of the four pollen parents, only crosses with the picoteeflowered S. vulgaris 'Sensation' produced seed. From 240 pollinations, 107 seeds were obtained; however, only one seed germinated and produced a viable seedling (H2013-150-01). Flow cytometry revealed H2013-150-01 to be a polyploid/ aneuploid with a $2 \mathrm{C}$ relative genome size of $5.65 \pm 0.02 \mathrm{pg}$ (Table 4). This genome size was significantly larger than any other seedling and is currently the largest reported in any lilac (Table 4) but is lower than expected for a tetraploid. In addition, the genome size of H2013-150-01 was larger than both its seed parent $[S$. vulgaris 'President Grévy' $(2 \mathrm{C}=4.85 \pm 0.00 \mathrm{pg})]$ and its pollen parent $[S$. vulgaris 'Sensation' $(2 \mathrm{C}=3.33 \pm 0.04 \mathrm{pg})]$. In a combined analysis on the flow cytometer, histograms for each parent as well as the hybrid were clearly distinguishable (Fig. 2).

Other studies have yielded similar results in crosses between diploids and triploids. Seedlings with genomes sizes surpassing their parents have been reported for diploid-triploid crosses in other woody plants including Pyrus (Phillips et al., 2016), Ulmus (Santamour, 1971), and Populus (Harder et al., 1976; Wang et al., 2010). Similar results have been found in herbaceous taxa including Miscanthus sinensis (Rounsaville et al., 2011) Rudbeckia (Palmer et al., 2009), Lilium (Lim et al., 2003; Marasek-Ciolakowska et al., 2014), Musa (Osuji et al., 1997), and Cucumis sativus (Diao et al., 2009). Most studies proposed sexual polyploidization via the union of unreduced gametes from one or both parents as the likely cause of the large seedling genomes. In the current study, H2013-150-01 resulted from an unreduced gamete from the diploid $S$. vulgaris

Table 4. Comparison of hybrid genome size from interploid and intraploid crosses in Syringa.

\begin{tabular}{|c|c|c|c|}
\hline Parent ploidy ${ }^{z}$ & $\operatorname{Cross}^{\mathrm{y}}$ & Accession no. ${ }^{x}$ & Relative $2 \mathrm{C}$ genome size $[\text { mean } \pm \text { SE }(\mathrm{pg})]^{\mathrm{w}}$ \\
\hline $3 x \times 2 x$ & S. vulgaris 'President Grévy' $\times S$. vulgaris 'Sensation' & H2013-150-01 & $5.65 \pm 0.02 \mathrm{a}$ \\
\hline \multirow{9}{*}{$2 x \times 3 x$} & & H2014-033-08 & $4.28 \pm 0.05 \mathrm{bc}$ \\
\hline & & H2014-033-05 & $4.25 \pm 0.03 \mathrm{bc}$ \\
\hline & & H2014-033-12 & $4.07 \pm 0.03 \mathrm{~cd}$ \\
\hline & & Н2014-033-09 & $4.02 \pm 0.13 \mathrm{cde}$ \\
\hline & & H2014-033-03 & $3.80 \pm 0.06 \mathrm{def}$ \\
\hline & & H2014-033-07 & $3.74 \pm 0.05$ ef \\
\hline & & H2014-033-10 & $3.74 \pm 0.04 \mathrm{f}$ \\
\hline & & Н2014-033-06 & $3.58 \pm 0.08 \mathrm{fg}$ \\
\hline & & H2014-033-11 & $3.41 \pm 0.03 \mathrm{gh}$ \\
\hline \multirow[t]{10}{*}{$2 x \times 2 x$} & $S$. vulgaris Tiny Dancer $\times S$. vulgaris 'Sensation' & H2014-032-17 & $3.30 \pm 0.05 \mathrm{~h}$ \\
\hline & & H2014-022-02 & $3.23 \pm 0.06 \mathrm{~h}$ \\
\hline & & H2014-022-04 & $3.16 \pm 0.04 \mathrm{~h}$ \\
\hline & S. $\times$ hyacinthiflora 'Old Glory' $\times S$. vulgaris 'Angel White' & H2014-024-16 & $3.27 \pm 0.08 \mathrm{~h}$ \\
\hline & & H2014-024-25 & $3.23 \pm 0.04 \mathrm{~h}$ \\
\hline & & H2014-024-27 & $3.23 \pm 0.02 \mathrm{~h}$ \\
\hline & & H2014-024-22 & $3.22 \pm 0.04 \mathrm{~h}$ \\
\hline & & H2014-024-03 & $3.20 \pm 0.03 \mathrm{~h}$ \\
\hline & S. vulgaris 'Sensation' $\times S$. vulgaris Tiny Dancer & H2014-027-08 & $3.22 \pm 0.03 \mathrm{~h}$ \\
\hline & & H2014-027-03 & $3.19 \pm 0.06 \mathrm{~h}$ \\
\hline
\end{tabular}

\footnotetext{
${ }^{\mathrm{z}}$ Ploidy of parent taxa including triploid by diploid $(3 x \times 2 x)$, diploid by triploid $(2 x \times 3 x)$, and diploid by diploid $(2 x \times 2 x)$ crosses.

${ }^{\mathrm{y}}$ Crosses among cultivars in series Syringa; seed parent listed first and pollen parent listed second.

${ }^{\mathrm{x}}$ Individual accessions in research population.

${ }^{\text {w } R e l a t i v e ~ 2 C ~ h o l o p l o i d ~ g e n o m e ~ s i z e s ; ~ m e a n s ~ s e p a r a t e d ~ u s i n g ~ T u k e y ' s ~ h o n e s t ~ s i g n i f i c a n t ~ d i f f e r e n c e ~(H S D) ~ t e s t ~ a t ~(~} \alpha=0.05$; minimum significant difference $=0.286$ ); means followed by the same letter are not significantly different.
} 


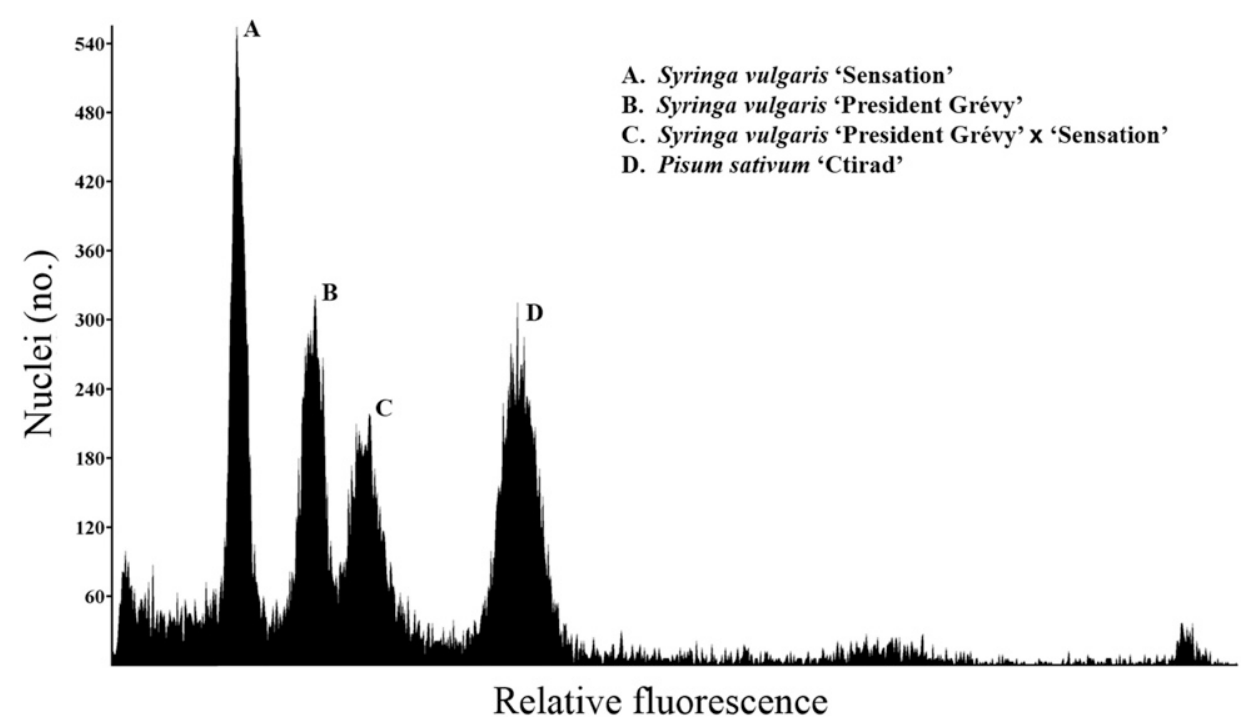

Fig. 2. Flow cytometry histogram of three taxa of Syringa vulgaris with an internal standard: (A) 'Sensation' (2C relative genome size $=3.33 \mathrm{pg})$; $(\mathbf{B})$ 'President Grévy' (2C relative genome size $=4.85 \mathrm{pg})$; (C) hybrid (H2013150-01) 'President Grévy' $\times$ 'Sensation' (2C relative genome size $=5.65 \mathrm{pg}$ ); (D) internal standard Pisum sativum 'Ctirad' (2C genome size $=8.76 \mathrm{pg})$.

'Sensation' (discussed below) and an aneuploid gamete from the triploid $S$. vulgaris 'President Grévy' that was $0.11 \mathrm{pg}$ below the expected haploid (1.5x) value. However, $S$. vulgaris 'Sensation' used in $2 x \times 2 x$ reciprocal crosses with $S$. vulgaris 'Tiny Dancer' failed to contribute unreduced gametes to produce triploid seedlings (Table 4).

As a pollen parent, the triploid $S$. vulgaris 'President Grévy' was used in crosses with $S$. vulgaris Blue Skies ${ }^{\circledR}, S$. vulgaris 'President Lincoln', and S. vulgaris 'Sensation' totaling 459 pollinations (Lattier and Contreras, 2017). Crosses with $S$. vulgaris Blue Skies ${ }^{\circledR}$ produced the only viable seed with 12 seedlings recovered from 135 pollinations (Lattier and Contreras, 2017). With the exception of one seedling (H2014-033-11), all hybrid seedlings varied in 2C genome size between the two parents, $S$. vulgaris Blue Skies ${ }^{\circledR}(3.44 \pm 0.03 \mathrm{pg})$ and $S$. vulgaris 'President Grévy' (4.85 \pm 0.00 pg) (Table 4). Relative 2C genome sizes of seedlings varied significantly from $3.41 \pm 0.03 \mathrm{pg}$ (H2014-033-11) to $4.35 \pm 0.03$ pg (H2014-033-01) (Table 4), the majority representing aneuploid genome sizes.

The estimations of chromosome numbers across aneuploid populations can be performed with knowledge of holoploid 2C genome sizes using Eq. [1]. Previous studies have produced models based on a holoploid genome size of a theoretical average, single chromosome based on parent genome sizes, and chromosome counts. Although some estimates of aneuploid chromosomes have been based solely on hypothetical chromosome size (Palmer et al., 2009), several studies have tested this model with root squashes and found most of their predictions to be concurrent with the true chromosome number or accurate within two to three chromosomes in Primula (Hayashi et al., 2009), Lilium (Lim et al., 2003), and Calluna (Behrend et al., 2015). Considering these previous studies and the relatively uniform chromosome size observed in lilac (Fig. 1), a simple linear model was used to predict chromosome number in the $2 x \times 3 x$ aneuploid population (Fig. 3).

Based on a linear model with an average chromosome size of $0.06 \mathrm{pg}$ (Fig. 3), the chromosome numbers in our aneuploid seedlings from $S$. vulgaris Blue Skies ${ }^{\circledR} \times S$. vulgaris 'President
Grévy' varied from 46 to 61 with an average of $54.3 \pm 1.4$ chromosomes. Chromosome numbers of triploid gametes can be deduced by subtracting the euploid chromosome number from the seedlings somatic chromosome numbers (Iorizzo et al., 2012). Assuming that $S$. vulgaris Blue Skies ${ }^{\circledR}$ consistently contributed haploid gametes with 23 chromosomes, S. vulgaris 'President Grévy' produced a range of aneuploid pollen from 23 to 38 chromosomes to progeny from this cross. Previous research in other crops has shown that triploids produce a higher percentage unreduced and/or aneuploid gametes than their diploid or tetraploid counterparts (Burton and Husband, 2001; Herben et al., 2016; Ramsey and Schemske, 1998). Viable aneuploid gametes have been described in plants such as $C$. sativus (Diao et al., 2009), Brassica (Brassica; Lu and Kato, 2001), and Tulipa (MarasekCiolakowska et al., 2014), yet other plants only tolerate euploid gametes as in Vaccinium corymbosum (Vorsa and Ballington, 1991). The resulting aneuploids from the $2 x \times 3 x$ lilac crosses were skewed slightly to the diploid cytotype compared with a theoretical bimodal distribution with an average of 57.5 chromosomes (Fig. 3). In the $3 x \times 2 x$ cross, S. vulgaris 'President Grévy' $\times S$. vulgaris 'Sensation', the triploid parent contributed 39 chromosomes $(2 n=7)$ as a seed parent. It is unclear if this slightly higher contribution from the triploid is due to combining with an unreduced gamete, the direction of the cross, or chance. Brandham (1982) reported a greater prevalence of aneuploidy over the range between diploid and triploid when the latter are females-presumably associated with endosperm balance number (discussed in later sections). However, with only a single seedling it is impossible to draw conclusions.

Although our seedling cytotypes varied from a random distribution of aneuploid cytotypes, lilac aneuploid segregation conflicts with the limited number of similar studies on $2 x \times 3 x$ crosses by being less concentrated at either euploid level (diploid or triploid). In lilies, these crosses resulted in all triploid or near-triploid seedlings derived from viable $2 n$ gametes from triploid male parents (Marasek-Ciolakowska et al., 2014). In Tulipa, $2 x \times 3 x$ crosses yielded a majority of diploid and near-diploid progeny with a small percent of neartriploids (Mizuochi et al., 2009). This same study found that the reciprocal cross in Tulipa yielded a binomial distribution of aneuploids, with the female triploid parent producing a wide range of fertile aneuploids (Mizuochi et al., 2009). Similar to lilies, $2 x \times 3 x$ crosses in Allium schoenoprasum (Levan, 1936) and $C$. sativus (Diao et al., 2009) resulted in diploids or neardiploids with a small percent of near-triploids; the reciprocal crosses yielded a wider range of aneuploids. Brandham (1982) reviewed a number of studies on interploid crosses and found that with very few exceptions the triploid parent generally produced gametes that were either haploid or diploid based on 


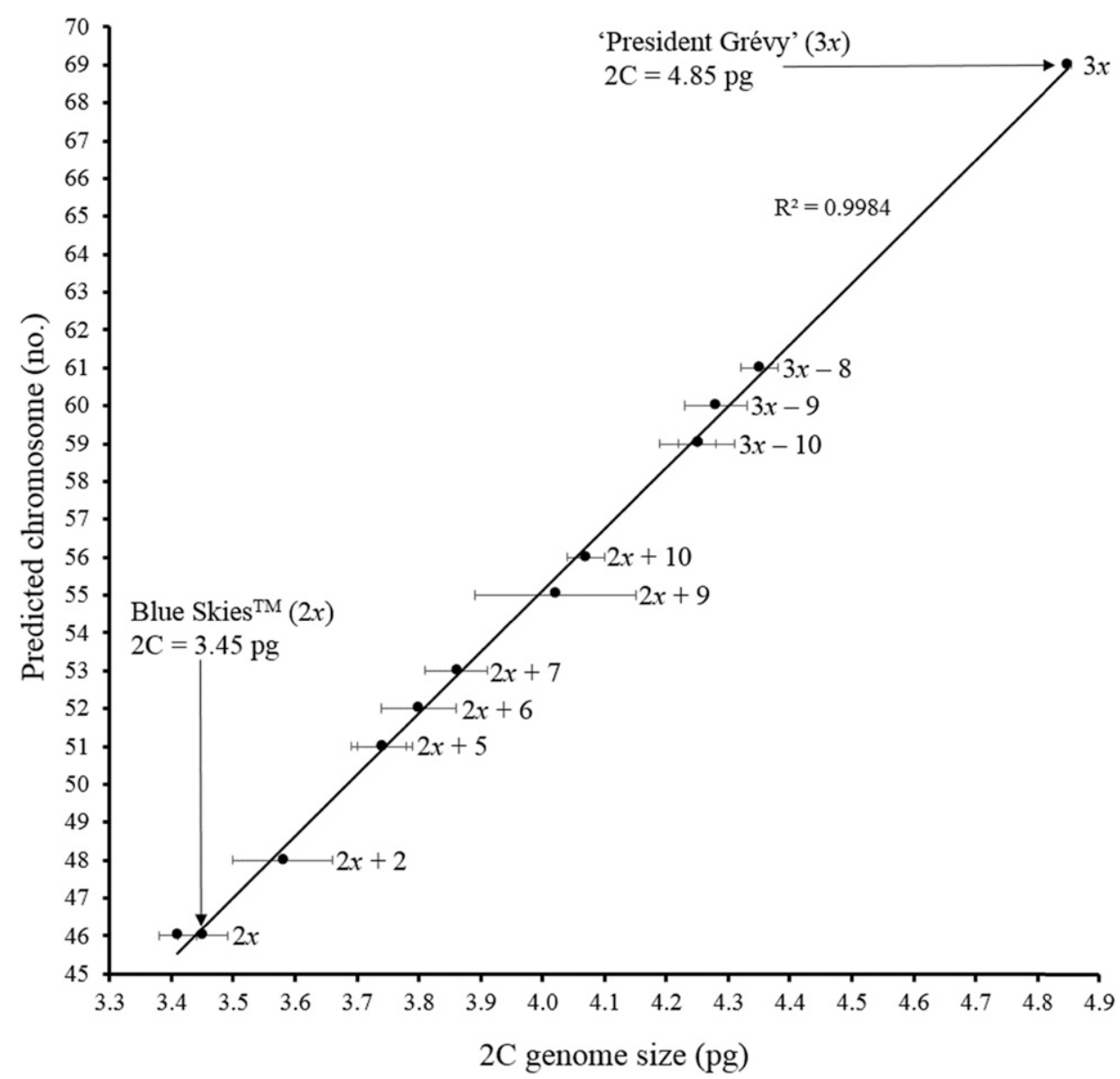

Fig. 3. Linear model of lilac $2 x \times 3 x$ aneuploid progeny with predicted chromosome number based on theoretical chromosome size of $0.061 \mathrm{pg}$ [(4.85 - $3.45 \mathrm{pg}) / 23$ chromosomes]. Parent taxa of aneuploid progeny: diploid female parent Syringa vulgaris 'Blue Skies' $(2 n=2 x=46)$ and triploid male parent $S$. vulgaris 'President Grévy' $(2 n=3 x=69)$. Linear model follows the formula: $y=16.224 x-9.7743$.

the ploidy of the other parent. Populus was a notable exception from other examples given, namely a considerably higher chromosome number than other taxa discussed. Populus $(x=19)$ is similar to Syringa $(x=23)$ in chromosome number and both are almost certainly of polyploid origin. This high chromosome number indicates that there is redundancy present that likely allows the survival of aneuploid gametes produced by triploids that are inviable in taxa with fewer base chromosomes.

The importance of embryo and endosperm cytotypes often plays a role in seedling cytotype segregation and has been studied at length in diploid and triploid crosses of potato (Solanum). A 2:1 maternal to paternal endosperm balance ratio must be maintained for successful hybridization in potato, preventing $2 x \times 3 x$ crosses and yielding progeny from $3 x \times 2 x$ that are skewed to near-triploid cytotypes (Carputo, 1999; Iorizzo et al., 2012). The origin of S. vulgaris 'President Grévy' could play a role in its fertility as a triploid parent. Allotriploids are rarely used in breeding because of their difficulty in chromosome pairing during meiosis; however, autotriploids can overcome problems with meiotic pairing to produce haploid to triploid gametes (Brandham, 1982; Hayashi et al., 2009; Kato et al., 2001). The history of wide hybridization and polyploid induction in lilac leaves the question open to the origins of triploid cytotypes. In addition, it remains unclear if meiotic abnormalities in gamete formation, preferential fertilization, or preferential embryo/endosperm survival skewed the distribution of aneuploid cytotypes. Our results may simply be due to the small sampling population of aneuploid seedlings resulting from the $2 x \times 3 x$ and $3 x \times 2 x$ crosses.

Despite the numerous pollinations and few resulting seedlings, all aneuploid lilacs appear to be healthy and vigorous after their first 2 years of growth (J.D. Lattier, personal observation). This conflicts with some studies which reported that aneuploid seedlings from diploid-triploid crosses were nonviable past initial germination exhibiting abnormal, stunted growth (Behrend et al., 2015; Osuji et al., 1997), and sometimes reverting to euploids after more than a year (Behrend et al., 2015). Although our aneuploids appear to grow as vigorously as their diploid counterparts, female fertility and pollen viability in the aneuploid population has not been investigated as the plants have yet to reach maturity during this study. However, flow cytometry was performed on the aneuploid population for more than 2 years after germination. While it cannot be assured that this aneuploid series will not stabilize at a euploid level (diploid or triploid), the fact that these plants have maintained aneuploid chromosome compliments for more than 2 years contrasts with previous studies and suggests that they may be stable.

UNREDUCED POLLEN. Stained pollen grains from four taxa of $S$. vulgaris were scored as viable and were measured for variability in diameter. Unstained pollen grains were negligible in all taxa, and pollen germination was not investigated. There were significant differences among taxa for pollen diameter $(P<0.0001)$ and every pairwise comparison between taxa was significant $(P<0.01)$. The largest average pollen grains were detected in $S$. vulgaris 'Sensation' (35.74 $\pm 0.16 \mu \mathrm{m})$, a measure likely overinflated by the presence of $8.5 \%$ unreduced pollen grains identified because of their increased volume (Fig. 4). This is the first report of unreduced $(2 n)$ pollen in lilac, but is not the first report in the Oleaceae. B-chromosomes and unreduced pollen have been reported in cultivars of Olea europaea (Sheidai et al., 2008). Syringa vulgaris 'Sensation' was also the only diploid observed to produce unreduced pollen, as $S$. vulgaris 'Ludwig Spaeth' and S. vulgaris 'Miss Ellen Willmott' produced only $1 n$ pollen (Fig. 4 ).

Because of its low fertility in crosses with the triploid seed parent S. vulgaris 'President Grévy' and the presence of an inflated genome size in the single viable seedling recovered (H2013-150-01), an unreduced pollen grain from $S$. vulgaris 'Sensation' likely contributed to the production of this single 


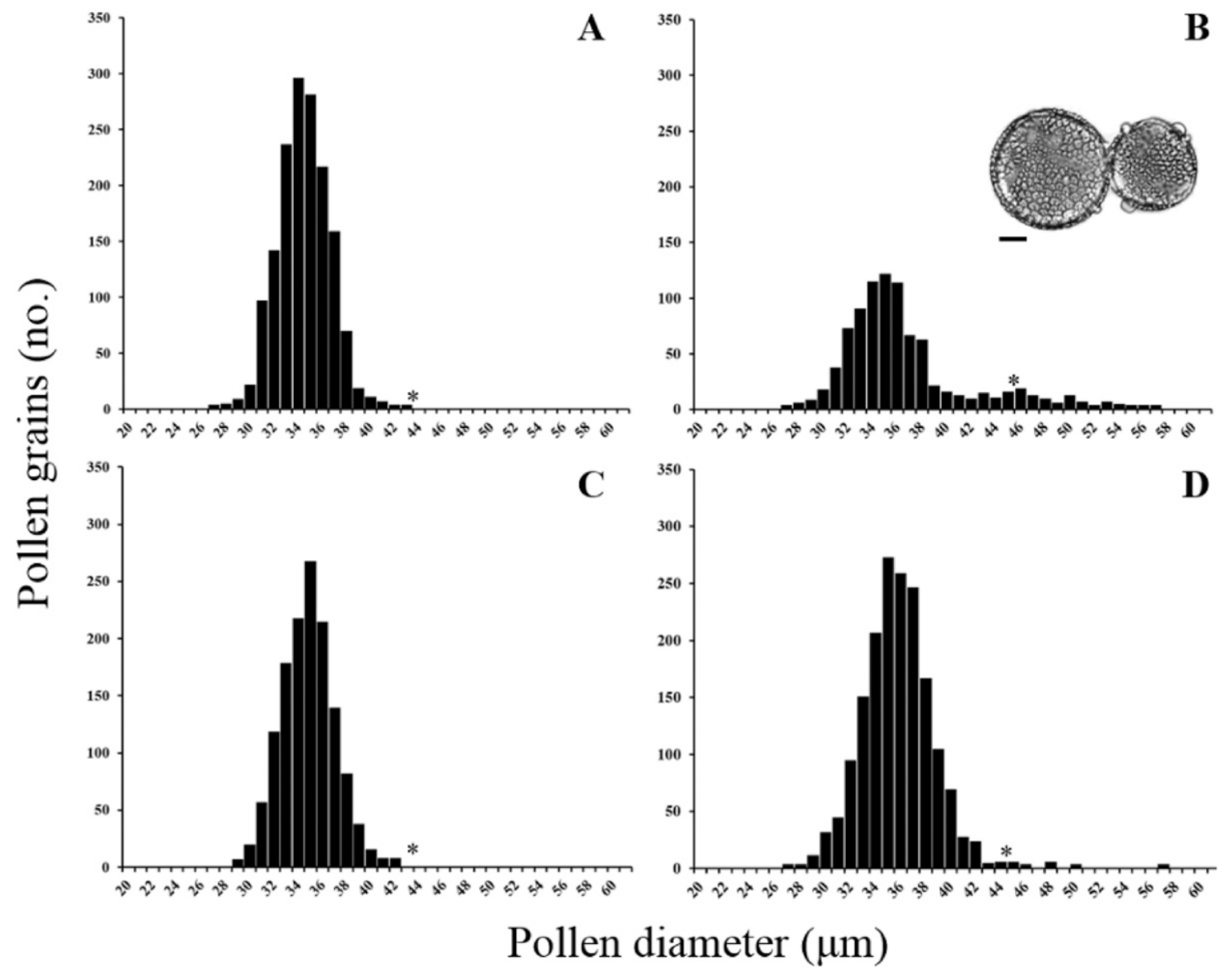

Fig. 4. Frequency distribution of viable pollen grain diameters of four cultivars of Syringa vulgaris. Regions to the right of asterisks were measured to be $26 \%$ larger than the mean and indicate unreduced gametes: (A) 'Ludwig Spaeth' ( $0 \%$ unreduced gametes); (B) 'Sensation' $(8.5 \%$ unreduced gametes) [insert: unreduced (left) and reduced (right) pollen grains stained with $2 \%$ acetocarmine and viewed at $\times 630$ magnification (scale bar $=10 \mu \mathrm{m})]$; (C) 'Miss Ellen Willmott' $(0 \%$ unreduced gametes); (D) 'President Grévy’ (0.6\% unreduced gametes).

polyploid/aneuploid progeny. It remains unclear whether the rare picotee flower mutation is in some way related to the production of an unreduced pollen grain, or if other diploids of S. vulgaris that were not included in the current study produce unreduced pollen at a similar rate as $S$. vulgaris 'Sensation'. Since an unreduced pollen grain resulted in the only viable seedling between these two cultivars, this may indicate the utility of an increased ploidy level for improving crosscompatibility in lilac.

Pollen from the double-flowering, triploid S. vulgaris 'President Grévy' proved difficult to obtain as many flowers simply did not produce viable anthers. Unreduced pollen grains $(0.6 \%)$ were detected in S. vulgaris 'President Grévy' out of 1689 grains measured. Although at a much lower percentage, $S$. vulgaris 'President Grévy' was the only parent besides $S$. vulgaris 'Sensation' to produce unreduced pollen in the current study, indicating some level of meiotic irregularities. Triploids are more likely to undergo irregularities during meiosis such as irregular chromosome pairing, supernumerary B chromosomes, laggard chromosomes, chromatin bridges, cytomixis, and out of plate chromosomes during metaphase I (Farco and Dematteis, 2014; Lavia et al., 2011). Triploids and resulting aneuploid progeny may prove to be sterile, yielding cultivars with reduced weediness and extended bloom times. Irregular meiosis during microspore development likely contributed to the poor performance of $S$. vulgaris 'President Grévy' as a seed parent and the subsequent aneuploidy seen in its viable seedlings (Fig. 3). Based on its aneuploid offspring when used as a male parent, $S$. vulgaris 'President Grévy' likely produces a range of aneuploid pollen. The average pollen grain diameter of $S$. vulgaris 'President Grévy' $(35.28 \pm 0.07 \mu \mathrm{m})$ was significantly larger than the two diploids that exhibited normal meiosis, $S$. vulgaris 'Ludwig Spaeth' $(33.96 \pm 0.05 \mu \mathrm{m})$ and $S$. vulgaris 'Miss Ellen Willmott' $(34.32 \pm 0.06 \mu \mathrm{m})$ from which no unreduced gametes were observed in a combined 2866 pollen grains (Fig. 4).

This study provides valuable information for future lilac breeding and informs a previous study on cross-compatibility among elite cultivars of lilac (Lattier and Contreras, 2017). In addition, this study contributes genome size and ploidy information to the growing database of angiosperm genome sizes, recommended by Galbraith et al. (2011). The discovery of three triploid lilacs, S. vulgaris 'Aucubaefolia', S. vulgaris 'Agincourt Beauty', and $S$. vulgaris 'President Grévy' lends evidence to previous reports of artificial tetraploid development and subsequent hybridization (Fiala and Vrugtman, 2008). However, no tetraploids were discovered among the research population. The discovery of high levels of aneuploidy in 
interploid hybrids indicates meiotic irregularities in pollen development of polyploid lilacs. Further cytological studies of pollen mother cells and meiotic analyses could contribute to understanding the complexities within developing gametes of taxa in the heavily hybridized series Syringa.

The development of an aneuploid series in $2 x \times 3 x$ crosses provides an avenue to develop a model for cytotype prediction in seedlings of interploid lilac hybrids. Future efforts to confirm initial predictions of aneuploid chromosome numbers will include chromosome counts on parent taxa, chromosome counts on a subset of aneuploids, and repollination of the parent genotypes to increase the number of seedlings in the aneuploid population. Further, aneuploids can be highly variable in morphology, including reduced vigor and can have greatly reduced fertility. This may be a detriment in breeding most crops, but could be an avenue for ornamental breeders to recover more compact, longer-blooming, and sterile cultivars. As the aneuploid population matures, plants will be compared for differences in gross morphology, and flowers will be compared for pollen viability and female fertility. Reanalyzing the genome sizes of this population will be necessary over subsequent years in light of previous reports of euploidization of woody aneuploids (Behrend et al., 2015).

The discovery of unreduced pollen in $S$. vulgaris 'Sensation' and subsequent production of a seedling from a $3 x \times 2 x$ cross with a larger genome than either parent, indicate that unreduced gametes or polyploidy may contribute to cross-compatibility in wide hybridization of lilac. Future work using high-throughput pollen screening by flow cytometry may reveal other cultivars with high levels of unreduced gametes. Wide hybridization with polyploids may reduce the impact of chromosome loss, which has been reported in previous cytological studies on lilac (Taylor, 1945 ) and the smallest genome recorded in the current study was a dissected-leaved, interspecific hybrid, S. xlaciniata. Identifying parents with unreduced pollen or generating autopolyploids in each lilac series may prove a valuable method for recovering viable progeny from wide hybridization in lilac. Interseries hybrids continue to be the most elusive quarry for lilac breeders, with only the pinnately compound $S$. pinnatifolia in the monophyletic series Pinnatifoliae proven to be a successful parent in crosses with $S$. oblata var. giraldii, $S$. vulgaris, $S . \times$ laciniata, and S. $\times$ hyacinthiflora (Pringle, 1981). Using S. pinnatifolia as well as an induced autopolyploids of cultivars proven to produce fruit and seed in interseries crosses (Lattier and Contreras, 2017) may spark a renaissance in the storied history of lilac breeding.

\section{Literature Cited}

Bai, C., W.S. Alverson, A. Follansbee, and D.M. Waller. 2012. New reports of nuclear DNA content for 407 vascular plant taxa from the United States. Ann. Bot. 110:1623-1629.

Behrend, A., A. Gluschak, A. Przybyla, and A. Hohe. 2015. Interploid crosses in heather (Calluna vulgaris). Sci. Hort. 181:162-167.

Bennett, M.D. and I.J. Leitch. 2011. Nuclear DNA amounts in angiosperms: Targets, trends and tomorrow. Ann. Bot. 107:467-590. Brandham, P.E. 1982. Inter-embryo competition in the progeny of autotriploid Aloineae (Liliaceae). Genetica 59:29-42.

Burton, T.L. and B.C. Husband. 2001. Fecundity and offspring ploidy in matings among diploid, triploid and tetraploid Chamerion angustifolium (Onagraceae): Consequences for tetraploid establishment. Heredity 87:573-582.

Carputo, D. 1999. Post-zygotic gametic selection due to endosperm balance number explains unusual chromosome numbers of $3 x \times 2 x$ progeny in Solanum. Sex. Plant Reprod. 12:27-31.
Chen, J.-T., Z.-S. Zhang, and D.-Y. Hong. 2009. A taxonomic revision of the Syringa pubescens complex (Oleaceae). Ann. Mo. Bot. Gard. 96:237-250.

Chen, Z.J. and Z. Ni. 2006. Mechanisms of genomic rearrangements and gene expression changes in plant polyploids. BioEssays 28:240252.

Contreras, R.N., M. Friddle, and J.D. Lattier. 2013. Relative fertility and ploidy levels of selected rose-of-sharon cultivars. Proc. Southern Nursery Assn. Res. Conf. 58:232-236.

Contreras, R.N., T.G. Ranney, and S.P. Tallury. 2007. Reproductive behavior of diploid and allotetraploid Rhododendron L. 'Fragrant Affinity'. HortScience 42:31-34.

Darlington, C.D. and A.P. Wylie. 1956. Chromosome atlas of flowering plants. Macmillan, New York, NY.

Diao, W.-P., S.-Y. Bao, B. Jiang, L. Cui, and J.-F. Chen. 2009. Primary trisomics obtained from autotriploid by diploid reciprocal crosses in cucumber. Sex. Plant Reprod. 22:45-51.

Farco, G.E. and M. Dematteis. 2014. Meiotic behavior and pollen fertility in trioploid and tetraploid natural populations of Campuloclinum macrocephalum (Eupatorieae, Asteraceae). Plant Syst. Evol. 300:1843-1852.

Fiala, J.L. and F. Vrugtman. 2008. Lilac: A gardener's encyclopedia. 2nd ed. Timber Press, Portland, OR.

Galbraith, D.W., J.L. Bennetzen, E.A. Kellogg, J.C. Pires, and P.S. Soltis. 2011. The genomes of all angiosperms: A call for a coordinated global census. J. Bot. 2011:1-10.

Green, P.S. 1995. Proposal to reject the name Syringa buxifolia Nakai (Oleaceae). Taxon 44:636.

Green, P.S. and M.C. Chang. 1995. Some taxonomic changes in Syringa L. (Oleaceae), including a revision of series Pubescentes. Novon 5:329-333.

Greilhuber, J. 1998. Intraspecific variation in genome size: A critical reassessment. Ann. Bot. 82:27-35.

Greilhuber, J., J. Doležel, M.A. Lysák, and M.D. Bennett. 2005. The origin, evolution and proposed stabilization of the terms 'genome size' and 'C-value' to describe nuclear DNA contents. Ann. Bot. 95:255-260.

Greilhuber, J., E.M. Temsch, and J.C.M. Loureiro. 2007. Nuclear DNA content measurement, p. 67-101. In: J. Doležel, J. Greilhuber, and J. Suda (eds.). Flow cytometry with plant cells: Analysis of genes, chromosomes and genomes. Wiley, Weinheim, Germany.

Harder, M.L., S. Verhagen, L. Winton, and D.W. Einspahr. 1976. Tetraploid aspen production using unreduced pollen from triploid males. For. Sci. 22:329-330.

Harlan, J.R. and J.M.J. deWet. 1975. On Ö. Winge and a prayer: The origins of polyploidy. Bot. Rev. 41:361-390.

Hayashi, M., J. Kato, H. Ohashi, and M. Mii. 2009. Unreduced $3 x$ gamete formation of allotriploid hybrid derived from the cross of Primula denticulata $(4 x) \times P$. rosea $(2 x)$ as a causal factor for producing pentaploid hybrids in the backcross with pollen of tetraploid P. denticulata. Euphytica 169:123-131.

Herben, T., P. Trávníček, and J. Chrtek. 2016. Reduced and unreduced gametes combine almost freely in a multiploidy system. Perspect. Plant Ecol. Evol. Syst. 18:15-22.

Iorizzo, M., R. Aversano, J.M. Bradeen, L. Frusciante, and D. Carputo. 2012. Fertilization fitness and offspring ploidy in $3 x \times 2 x$ matings in potato. Plant Biosyst. 146:317-321.

Kato, J., R. Ishikawa, and M. Mii. 2001. Different genetic combinations in inter-section hybrids obtained from the crosses between Primula sieboldii (section Cortusoides) and P. obconica (section Obconicolisteri) by the embryo rescue technique. Theor. Appl. Genet. 102:1129-1135.

Kim, K.-J. and R.K. Jansen. 1998. A chloroplast DNA phylogeny of lilacs (Syringa, Oleaceae): Plastome groups show strong correlations with crossing groups. Amer. J. Bot. 85:1338-1351.

Lattier, J.D., H. Chen, and R.N. Contreras. 2017. Improved method of enzyme digestion for root tip cytology. HortScience 52:10291032 . 
Lattier, J.D. and R.N. Contreras. 2017. Intraspecific, interspecific, and interseries cross-compatibility in lilac. J. Amer. Soc. Hort. Sci. 142:279-288.

Lattier, K.S. 2016. Inducing and evaluating phenotypic and cytometric variation in landscape plants: Observations from Acer, Ornithogalum, and Penstemon. Oregon State Univ., Corvallis, OR, MS Diss.

Lavia, G.I., A.M. Ortiz, G. Robledo, A. Fernández, and G. Seijo. 2011. Origin of triploid Arachis pintoi (Leguminosae) by autopolyploidy evidenced by FISH and meiotic behaviour. Ann. Bot. 108:103-111.

Levan, A. 1936. Different results in reciprocal crosses between diploid and triploid Allium schoenoprasum L. Nature 138:508.

Li, J., J.H. Alexander, and D. Zhang. 2002. Paraphyletic Syringa (Oleaceae): Evidence from sequences of nuclear and ribosomal DNA ITS and ETS regions. Syst. Bot. 27:592-597.

Li, J., B. Goldman-Huertas, J. DeYoung, and J. Alexander, III. 2012. Phylogenetics and diversification of Syringa inferred from nuclear and plastid DNA sequences. Castanea 77:82-88.

Lim, K.-B., M.S. Ramanna, E. Jacobsen, and J.M. van Tuyl. 2003. Evaluation of $\mathrm{BC}_{2}$ progenies derived from $3 x-2 x$ and $3 x-4 x$ crosses of Lilium hybrids: A GISH analysis. Theor. Appl. Genet. 106:568-574.

$\mathrm{Lu}, \mathrm{C}$. and M. Kato. 2001. Fertilization fitness and relation to chromosome number in interspecific progeny between Brassica napus and B. rapa: A comparative study using natural and resynthesized B. napus. Breed. Sci. 51:73-81.

Marasek-Ciolakowska, A., S. Xie, P. Arens, and J.M. van Tuyl. 2014. Ploidy manipulation and introgression breeding in darwin hybrid tulips. Euphytica 198:389-400.

Miller, G.N. 1955. The genus Fraxinus, the ashes, in North America, north of Mexico. Cornell Univ. Agr. Expt. Sta. Memoirs 335:1-64.

Mizuochi, H., H. Matsuzaki, T. Moue, and K. Okazaki. 2009. Diploid endosperm formation in Tulipa spp. and identification of a $1: 1$ maternal-to-paternal genome ratio in endosperms of $T$. gesneriana $\mathrm{L}$. Sex. Plant Reprod. 22:27-36.

Nesom, G.L. 2010. Fraxinus biltmoreana and Fraxinus smallii (Oleaceae), forest trees of the eastern United States. Phytoneuron 51:1-30.

Olsen, R.T., T.G. Ranney, and Z. Viloria. 2006. Reproductive behavior of induced allotetraploid $\times$ Chitalpa and in vitro embryo culture of polyploid progeny. J. Amer. Soc. Hort. Sci. 131:716-724.

Olszewska, M.J. and R. Osiecka. 1984. Relationship between 2C DNA content, systematic position and level of DNA endoreplication during differentiation of root parenchyma in dicot shrubs and trees. Comparison with herbaceous species. Biochem. Physiol. Pflanz. 179:641-657.

Osuji, J.O., D. Vuylsteke, and R. Ortiz. 1997. Ploidy variation in hybrids from interploid $3 x \times 2 x$ crosses in Musa. Tropicultura 15:37-39.

Palmer, I.E., T.G. Ranney, N.P. Lynch, and R.E. Bir. 2009. Crossability, cytogenetics, and reproductive pathways in Rudbeckia subgenus Rudbeckia. HortScience 44:44-48.

Parris, J.K., T.G. Ranney, H.T. Knap, and W.V. Baird. 2010. Ploidy levels, relative genome sizes, and base pair composition in Magnolia. J. Amer. Soc. Hort. Sci. 135:533-547.

Phillips, W.D., T.G. Ranney, D.H. Touchell, and T.A. Eaker. 2016. Fertility and reproductive pathways of triploid flowering pears (Pyrus sp.). HortScience 51:968-971.

Pringle, J.S. 1981. A review of attempted and reported interseries and intergeneric hybridization in Syringa (Oleaceae). Baileya 20:49-91.
Purdue University Cytometry Laboratories. 2014. Cytospec Version 7. 12 July 2017. <http://www.cyto.purdue.edu/Purdue_software>.

Ramsey, J. and D.W. Schemske. 1998. Pathways, mechanisms, and rates of polyploid formation in flowering plants. Annu. Rev. Ecol. Syst. 29:467-501.

Ranney, T.G. 2006. Polyploidy: From evolution to new plant development. Proc. Intl. Plant Propagators Soc. 56:604-607.

Rose, J.B., J. Kubba, and K.R. Tobutt. 2000. Chromosome doubling in sterile Syringa vulgaris $\times S$. pinnatifolia hybrids by in vitro culture of nodal explants. Plant Cell Tissue Organ Cult. 63:127-132.

Rothleutner, J.J. 2014. Development of autopolyploid Syringa reticulata subsp. pekinensis for breeding. Acta Hort. 1055:213-217.

Rounsaville, T.J. and T.G. Ranney. 2010. Ploidy levels and genome sizes of Berberis L. and Mahonia Nutt. species, hybrids, and cultivars. HortScience 45:1029-1033.

Rounsaville, T.J., D.H. Touchell, and T.G. Ranney. 2011. Fertility and reproductive pathways in diploid and triploid Miscanthus sinensis. HortScience 46:1353-1357.

Santamour, F.S., Jr. 1962. The relation between polyploidy and morphology in white and biltmore ashes. Bull. Torrey Bot. Club 89:228-232

Santamour, F.S., Jr. 1971. A tripoid elm (Ulmus pumila $\times$ U. rubra) and its aneuploid progeny. Bull. Torrey Bot. Club 98:310-314.

Sax, K. 1930. Chromosome number and behavior in the genus Syringa. J. Arnold Arbor. 11:7-14

Shearer, K. and T.G. Ranney. 2013. Ploidy levels and relative genome size of species, hybrids, and cultivars of dogwood (Cornus spp.). HortScience 48:825-830.

Sheidai, M., H. Parsian, S. Vaezi-Joze, and Z. Noormohammadi. 2008. Chromosome pairing and chiasma formation in some olive (Olea europaea L.) cultivars from Iran. Cytologia 73:269-274.

Siljak-Yakovlev, S., F. Pustahija, E.M. Šolić, F. Bogunić, E. Muratović, N. Bašić, O. Catrice, and S.C. Brown. 2010. Towards a genome size and chromosome number database of Balkan flora: $C$-values in 343 taxa with novel values for 242. Adv. Sci. Lett. 3:190213

Soltis P.S. and D.E. Soltis (eds.). 2012Polyploidy and genome evolution. Springer-Verlag, Berlin, Germany.

Stebbins, Jr., G.L., 1940. The significance of polyploidy in plant evolution. Am. Nat. 74:54-66.

Taylor, H. 1945. Cyto-taxonomy and phylogeny of the Oleaceae. Brittonia 5:337-367.

Vorsa, N. and J.R. Ballington. 1991. Fertility of triploid highbush blueberry. J. Amer. Soc. Hort. Sci. 116:336-341.

Wallander, E. and V.A. Albert. 2000. Phylogeny and classification of Oleaceae based on $r p s 16$ and $\operatorname{trnL}-F$ sequence data. Amer. J. Bot. 87:1827-1841.

Wang, J., X. Kang, and Q. Zhu. 2010. Variation in pollen formation and its cytological mechanism in an allotriploid white poplar. Tree Genet. Genomes 6:281-290.

Zonneveld, B.J.M. and G.D. Duncan. 2010. Genome sizes of Eucomis L'Hér. (Hyacinthaceae) and a description of the new species Eucomis grimshawii G.D. Duncan \& Zonneveld. Plant Syst. Evol. 284:99109.

Zonneveld, B.J.M., I.J. Leitch, and M.D. Bennett. 2005. First nuclear DNA amounts in more than 300 angiosperms. Ann. Bot. 96:229-244. 